\title{
La loi fédérale sur l'analyse génétique humaine (LAGH) doit être envisagée sous un aspect critique
}

\author{
La loi fédérale sur l'analyse génétique humaine (LAGH ) entre en vigueur \\ le $1^{\text {er }}$ avril 2007. Cette dernière entraîne diverses modifications du «Rapport \\ de l'examen médical».
}

Peter A. Suter

Président de la Commission pour l'appréciation médicale des risques de l'Association Suisse d'Assurances ASA
Association Suisse d'Assurances ASA C.F. Meyer-Strasse 14

Case postale 4288

CH-8022 Zurich

Tél. +41442082828

Fax +41442082800

\begin{abstract}
Introduction
En 2004 déjà, le Parlement a approuvé la nouvelle loi sur l'analyse génétique humaine. Par l'intermédiaire du Conseil fédéral, cette loi entre définitivement en vigueur le $1^{\text {er }}$ avril 2007. La section: «Analyses génétiques dans le domaine de l'assurance» ne contient pas de dispositions d'exécution spécifiques. Toutefois, cette nouvelle réglementation légale influence le déroulement pratique de l'établissement d'une proposition dans le domaine de l'assurance de personnes. C'est pourquoi la «Commission pour l'appréciation des risques médicaux» de l'Association Suisse d'Assurances (ASA) s'est penchée sur la question. Le groupe de travail mis sur pied a, d'une part, adapté à la nouvelle situation le «Rapport de l'examen médical» avec la parution de l'édition 2006. D'autre part, ce groupe de travail a également émis des recommandations à l'intention des compagnies membres de l'ASA.
\end{abstract}

\section{Réglementation légale}

Les articles légaux d'importance pour les assureurs vie sont brièvement évoqués ici. Il s'agit des articles ci-après:

- Art. 3: Définitions

- Art. 26: Interdiction d'exiger une analyse

- Art. 27: Interdiction d'exiger ou d'utiliser les résultats d'une analyse déjà effectuée

- Art. 28: Autorisation d'exiger ou d'utiliser les résultats d'une analyse génétique présymptomatique déjà effectuée.

Il faut relever spécialement ici la distinction à faire entre les analyses génétiques présymptomatiques et les analyses génétiques prénatales. Pour le médecin traitant, il est important de savoir que

- les analyses génétiques ou les questions relatives à leurs résultats ne sont pas interdites par la loi;
- exiger ou utiliser les résultats d'une analyse génétique présymptomatique déjà effectuée n'est possible qu'à titre exceptionnel et en observant de strictes façons de procéder;

- d'une manière générale, les analyses génétiques prénatales ne peuvent pas être utilisées.

La teneur exacte des articles mentionnés, comme la LAGH dans son ensemble, peut être consultée à l'adresse www.svv.ch (Publications spécialisées/ Droit des assurances privées).

\section{Le point de vue des assureurs vie}

De l'avis des assureurs vie, la nouvelle loi fédérale doit être envisagée sous un aspect critique. En effet, c'est la première fois qu'une loi limite la possibilité de l'évaluation des risques dans le domaine de l'assurance privée et s'accommode d'une asymétrie de l'information. Il peut en résulter une situation où la personne assurée dispose d'informations relatives au risque, mais auxquelles, du fait de la loi, l'assureur vie ne doit ou ne peut pas avoir accès. Par ailleurs, il faut aussi relever que les résultats d'analyses génétiques déjà effectuées peuvent être utilisés dans certains cas pour apprécier le risque. De plus, en comparaison internationale, nous sommes soumis à des dispositions plus restrictives et, à contrario, à d'autres dispositions plus libérales.

\section{Nouveau Rapport de l'examen médical} des sociétés suisses d'assurances sur la vie L'édition 2006 du Rapport de l'examen médical présente deux modifications essentielles, ainsi que diverses adaptations mineures uniquement destinées à en améliorer la qualité. 


\section{Obligation d'informer}

Une grande importance est accordée à l'obligation d'informer. C'est la raison pour laquelle, en guise d'introduction, le Rapport de l'examen médical attire l'attention sur ce qui est autorisé et ce qui est interdit en relation avec les analyses génétiques et leurs résultats. Pour le médecin examinateur, il est important de savoir qu'aucun résultat d'analyses génétiques présymptomatiques ne peut être mentionné.

\section{Extrait:}

La loi interdit aux assureurs de demander des résultats d'analyses génétiques prénatales. Par contre, si certaines conditions sont remplies, ils peuvent demander les résultats d'analyses génétiques présymptomatiques (analyses effectuées pour déterminer s'il $\mathrm{y}$ a des prédispositions à une maladie, avant que des symptômes ne soient apparus). Si ces conditions sont remplies, la clarification a lieu au moyen d'un formulaire séparé. De ce fait, de tels résultats d'analyses ne doivent pas être mentionnés dans le présent questionnaire. De même, des résultats communiqués volontairement ne peuvent pas être utilisés par les assureurs.

Des analyses génétiques effectuées à des fins de diagnostic, autrement dit pour faire la lumière sur des symptômes de maladie déjà constatables, ne sont pas concernées par la disposition légale et doivent être déclarées.

\section{Anamnèse familiale}

La question portant sur l'anamnèse familiale a été modifiée en fonction de son importance médicale. Ceci notamment parce que les questions portant sur des analyses-tests de cette nature ne sont, en règle générale, pas autorisées.

Concrètement, et c'est nouveau, les questions ne portent que sur des maladies apparues avant l'âge de 55 ans. Il ne faut plus indiquer la personne qui a été atteinte par cette maladie, mais, seconde nouveauté, uniquement chez combien de personnes elle s'est présentée.

Extrait:

Est-ce que chez vos parents, frères et sœurs, grandsparents, des troubles neurologiques, maladies cardiaques, attaques cérébrales, diabètes sucrés, cancers ou maladies héréditaires sont apparus avant l'âge de 55 ans?

\section{Autres adaptations}

A titre d'exemple, on relèvera ici que le tour de taille et le tour de hanches doivent maintenant être indiqués.

\section{Manière de procéder dans la pratique}

S'il est satisfait à toutes les conditions légales et si cela paraît indiqué pour l'appréciation du risque, il est possible, par le biais d'un processus compliqué à plusieurs niveaux - ainsi le veut la loi - de se renseigner sur le résultat d'une analyse génétique présymptomatique.

Le résultat de l'analyse ne peut toutefois être exigé que s'il paraît fiable et déterminant pour le calcul des primes. A cet effet il est, pour chaque cas individuel, procédé avec les services spécialisés d'une importante compagnie de réassurance à un ajustement en fonction de l'évolution actuelle. Dans un premier temps, il est demandé à la personne assurée - à l'aide d'un questionnaire additionnel - s'il a déjà été procédé à une analyse génétique. Le cas échéant, le médecin concerné doit être indiqué. Toutefois, les résultats n'ont pas à être communiqués. Ce questionnaire additionnel doit être envoyé au médecinconseil de la compagnie et ce dernier décide s'il y a lieu de prendre contact avec le médecin examinateur et de demander les résultats de l'analyse génétique présymptomatique. Tout ceci bien entendu uniquement si toutes les conditions selon la LAGH sont remplies.

On trouvera à l'art. 28 de la LAGH des indications détaillées sur ce processus. A signaler en outre la publication Infomed - Bulletin sur l'assurance vie, édition 2006/1 consacrée à la question des analyses génétiques humaines. L'envoi à titre de supplément au Bulletin des médecins suisses no 26 a été fait en juin 2006. Ladite publication est disponible sous forme électronique à l'adresse www.svv.ch (Publications spécialisées / Infomed).

\section{Remarques finales}

Les assureurs vie ne seront certainement pas encore submergés par cette question réellement complexe dans les mois à venir. Reste qu'à moyen et long terme la question va revêtir toute son importance. Les assureurs vie mettront assurément tout en œuvre afin de satisfaire aux multiples intérêts en jeu et, si nécessaire en raison des premières expériences, certains points (par exemple limites de sommes) seront remis sous la loupe. 